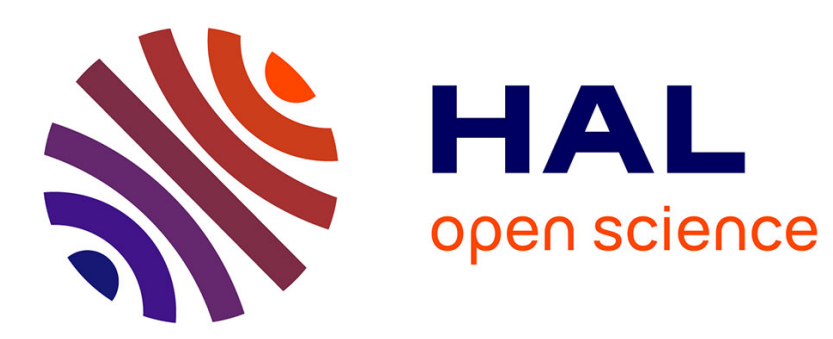

\title{
On the field dependence of the spin glass susceptibility peak
}

\author{
B. Barbara, A. P. P Malozemoff, S. E. E Barnes
}

\section{To cite this version:}

B. Barbara, A. P. P Malozemoff, S. E. E Barnes. On the field dependence of the spin glass susceptibility peak. Journal of Applied Physics, 1984, 55 (6), pp.1655 - 1657. 10.1063/1.333432 . hal-01660409

\section{HAL Id: hal-01660409 \\ https://hal.science/hal-01660409}

Submitted on 10 Dec 2017

HAL is a multi-disciplinary open access archive for the deposit and dissemination of scientific research documents, whether they are published or not. The documents may come from teaching and research institutions in France or abroad, or from public or private research centers.
L'archive ouverte pluridisciplinaire HAL, est destinée au dépôt et à la diffusion de documents scientifiques de niveau recherche, publiés ou non, émanant des établissements d'enseignement et de recherche français ou étrangers, des laboratoires publics ou privés. 


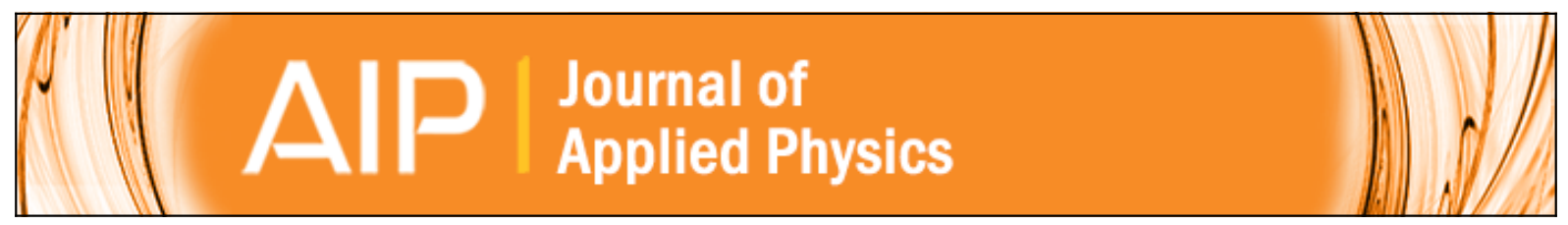

\section{On the field dependence of the spin glass susceptibility peak}

B. Barbara, A. P. Malozemoff, and S. E. Barnes

Citation: Journal of Applied Physics 55, 1655 (1984); doi: 10.1063/1.333432

View online: http://dx.doi.org/10.1063/1.333432

View Table of Contents: http://scitation.aip.org/content/aip/journal/jap/55/6?ver=pdfcov

Published by the AIP Publishing

\section{Articles you may be interested in}

Anisotropy Dependence of the Chiral Susceptibility in Canonical Spin Glasses

AIP Conf. Proc. 850, 1117 (2006); 10.1063/1.2355094

Fielddependent susceptibility aging in CuMn spin glasses

J. Appl. Phys. 76, 6192 (1994); 10.1063/1.358347

Lowfield susceptibility of GdAl spin glass: Coolingrate dependence and field dependence of peak temperature J. Appl. Phys. 57, 3389 (1985); 10.1063/1.335105

A comparison of the systematics of the spinglass susceptibility peaks in $\mathrm{P} d \mathrm{Mn}$ with an effectivefield model J. Appl. Phys. 57, 3447 (1985); 10.1063/1.335072

Temperature dependence of the nonlinear susceptibility in spin glasses

J. Appl. Phys. 53, 7693 (1982); 10.1063/1.330184

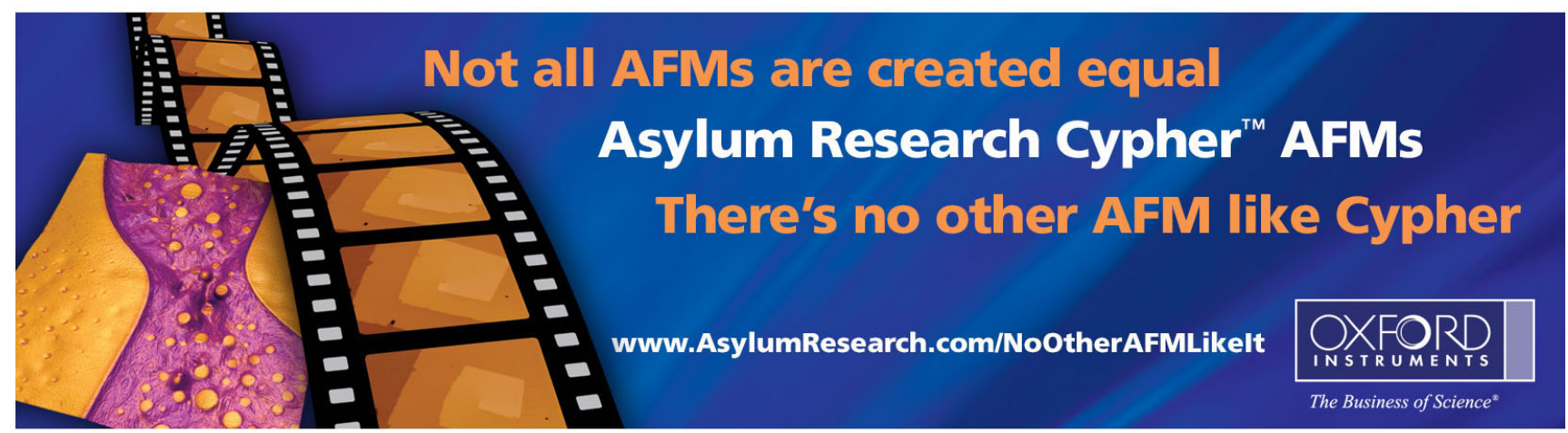




\section{On the field dependence of the spin glass susceptibility peak}

B. Barbara

Laboratoire Louis Néel, CNRS, Grenoble 166X 38042, Cedex, France

A. P. Malozemoff

IBM Thomas J. Watson Research Center, Yorktown Heights, New York 10598

S. E. Barnes

University of Miami, Physics Department, Coral Gables, Florida 33124

In amorphous GdAl spin glass, as in a number of other spin glasses, the susceptibility cusp first increases, then decreases in temperature with increasing field. An explanation for this behavior is found in terms of a competition between the noncritical linear susceptibility and the critical nonlinear susceptibility.

PACS numbers: $75.50 . \mathrm{Kj}, 75.40 . \mathrm{Bw}$

Early lore about spin glasses held that the temperature of the susceptibility cusp shifts down with increasing magnetic field. Nevertheless, even the early results of Cannella and Mydosh ${ }^{1}$ on AuFe spin glasses showed the opposite tendency. More recently, our own studies ${ }^{2}$ of the dc susceptibility of CuMn and amorphous GdAl spin glasses also showed the peak temperature shifting up with field. Yeshurun ${ }^{3}$ found a similar effect in an amorphous FeMnPBAl spin glass. Results of a more complete study ${ }^{4}$ on our amorphous GdAl sample ${ }^{5}$ are shown as circles in Fig. 1(a). The peak temperature $T_{P}$ first increases with field $H$, but later decreases as the peak broadens. In terms of a reduced temperature $t=\left(T-T_{G}\right) / T_{G}$, with the zero-field transition temperature $T_{G}$ at $15.5 \mathrm{~K}$ for our sample, the initial shift with field follows $t=0.0061 H^{0.50}$.

Ours is de equilibrium data, while most other studies have been done with ac techniques, which introduce nonequilibrium effects. Aside from our $\mathrm{GdAl}$ and $\mathrm{CuMn}$ results, ${ }^{2}$ and those of Yeshurun, ${ }^{3}$ the only other detailed dc equilibrium study of the field dependence is that of Chamberlin et al. ${ }^{6}$ on a AgMn spin glass, where $T_{P}$ decreases with $H$. We interpret the present results as indicating that both behaviors are possible, depending on the material, and a satisfactory theory should explain why.

In this paper we show how a non-mean-field scaling theory can explain the remarkable behavior of Fig. 1. As described in more detail elsewhere, ${ }^{4,7}$ such a theory predicts a singular contribution $\chi_{\text {sing }}$ to the equilibrium susceptibility within a critical region near and above the phase transition temperature $T_{G}$. In terms of the reduced temperature $t=\left(T-T_{G}\right) T_{G}$, scaling exponents $\beta, \phi, \delta$, and $\gamma$, and a scaling function $g$, the singular susceptibility has the form

$$
\begin{aligned}
& \chi_{\text {sing }}=t^{\beta} g\left(H^{2} / t^{\phi}\right), \\
& g(x) \rightarrow g_{0}-g_{1} x, \text { for } x \rightarrow 0, \\
& g(x) \rightarrow-g_{2} x^{1 / \delta}, \text { for } x \rightarrow \infty, \\
& \delta=\phi / \beta, \\
& \gamma=\phi-\beta=\beta(\delta-1),
\end{aligned}
$$

where $g_{0}, g_{1}$, and $g_{2}$ are constants. Therefore, for $H$ fixed, $\chi_{\text {sing }}$ has the limits

$$
\begin{aligned}
& \chi_{\text {sing }} \rightarrow g_{0} t^{\beta}-g_{1} H^{2} t^{-\gamma}, \text { for } t \rightarrow \infty, \\
& \rightarrow-g_{2} H^{2 / \delta}, \text { for } t \rightarrow 0 .
\end{aligned}
$$

Plots of $\chi_{\text {sing }} / H^{2 / \delta}$ vs $t / H^{2 / \phi}$ [which is an equivalent way to test Eq. (1)] have indeed shown scaling. ${ }^{7}$ A specific form for $\chi_{\text {sing }}$ which is consistent with Eqs. (1) and (2) and which smoothly interpolates between the limits is ${ }^{8}$

$$
\chi_{\text {sing }}=-g_{1} H^{2 / \delta} /\left[\left(t / H^{2 / \phi}\right)+\left(g_{1} / g_{2}\right)^{1 / \gamma}\right]^{\gamma} .
$$

In the low-field limit, this form is regular in $H^{2}$ as indicated by Eq. (2), and in the high-field or low-temperature limit it is regular in $t$. Both these properties are important in achieving an accurate fit to the data, to be described below. It should also be mentioned that the $g_{0}$ term of Eq. (2) is ignored in Eq. (3), because it can be shown to be negligibly small in fits to the data. $^{9}$

Next we ask, can the behavior of the susceptibility peak be understood from Eqs. (1) or (3)? Differentiating Eq. (1), we find the condition for an extremum:

$$
\beta g(x)=\phi x g^{\prime}(x), \quad x=h^{2} t-\phi .
$$

Any real solution of this equation corresponds to an extremum line following $t \propto h^{2 / \phi}$. That is, such a line is governed by the same exponent $\phi$ which describes the crossover between the low or high temperature limits of Eq. (1). However, the existence of such an extremum line depends on the specific form of $g$. We have recently argued elsewhere ${ }^{4}$ that on physical grounds, $g_{0}, g_{1}$, and $g_{2}$ should all be positive for $t>0$. In this case, it is easy to see from Eqs. (3) and (4) that there is no solution at all for $t>0$. This conclusion is in contradiction to the observed tendency of the peak to shift to higher $t$ in our sample.

The resolution of this difficulty, we believe, lies in recognizing the importance of regular (nonsingular) contributions to the susceptibility. From a physical point of view, such contributions represent spins or clusters of spins which do not participate in the critical fluctuations. From the point of view of analyzing experimental susceptibility data, they play a more important role in spin glasses than in ferromagnets for two reasons. (1) The singular behavior for a ferromagnet occurs in the linear susceptibility, while for a spin glass it occurs in the nonlinear susceptibility. Determination of the nonlinear susceptibility is more subtle, requiring proper subtraction of the linear terms and greater experimental 


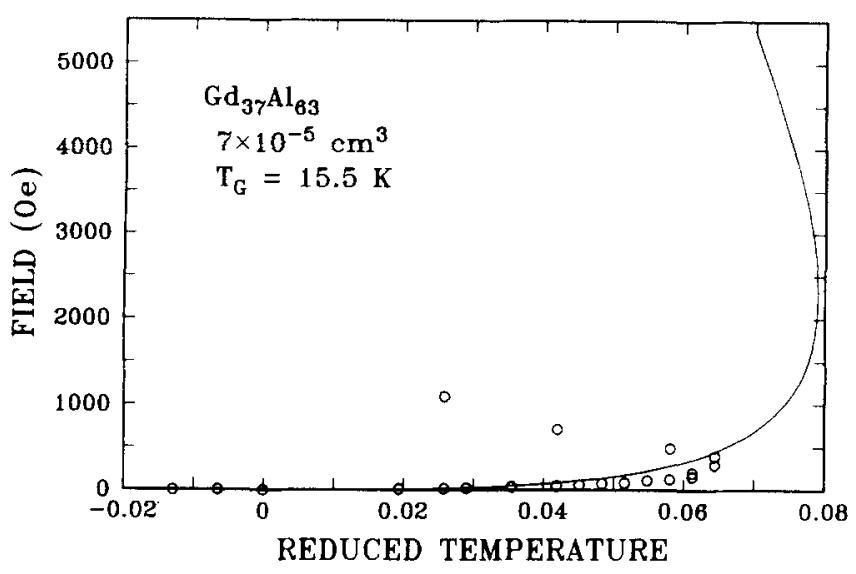

(a)

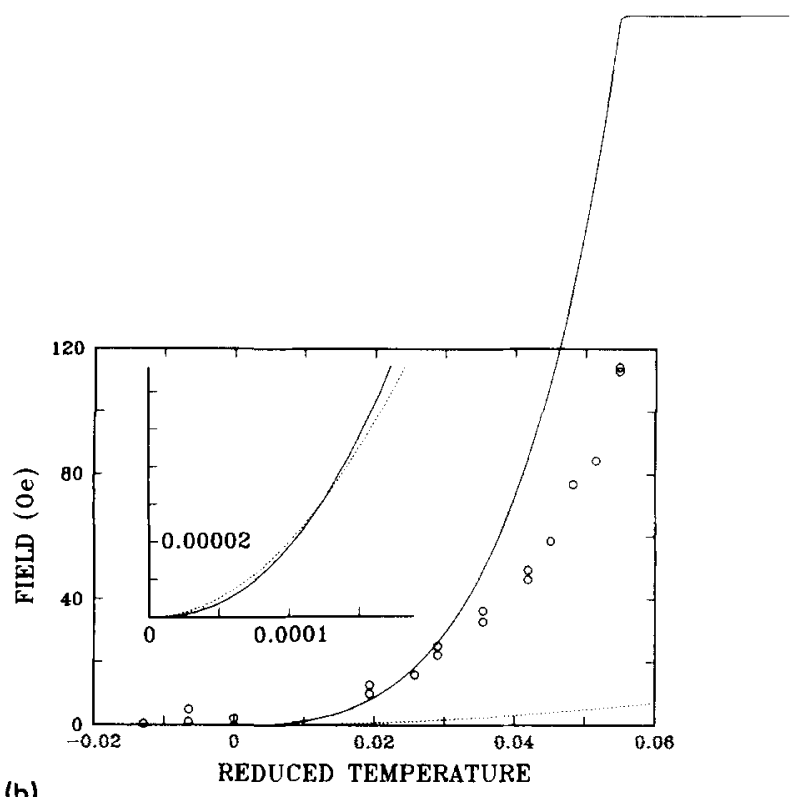

FIG. 1. Susceptibility peak position (points) for $\mathrm{Gd}_{37} \mathrm{Al}_{63}$ amorphous spin glass. Parts (a) and (b) and the insert show different scales. The reduced temperature is $(T-15.5 \mathrm{~K}) / 15.5 \mathrm{~K}$. The solid line is the predicted line of maxima, from Eq. (3) and fit 1 of the table. The dotted line is the crossover line $t=\left(g_{1} / g_{2}\right)^{1 / \gamma} H^{2 / \phi}$.

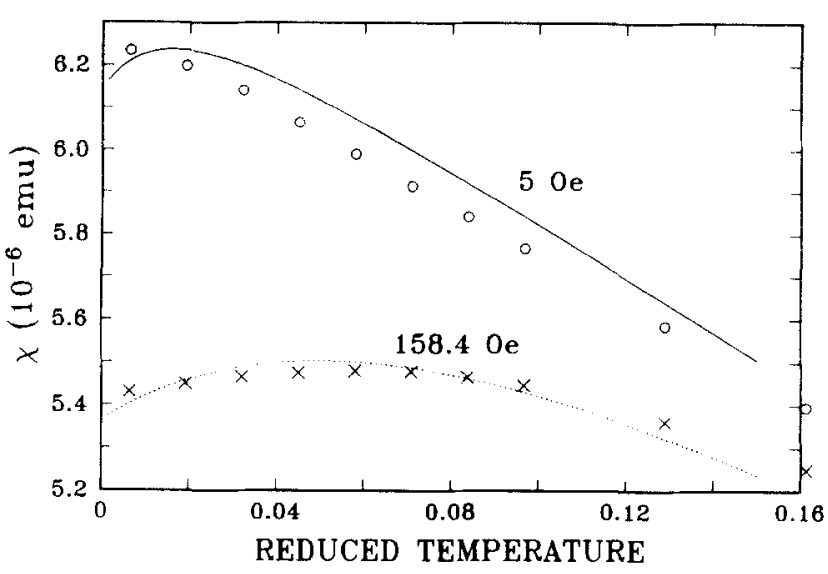

FIG. 2. Susceptibility vs reduced temperature (points) for the GdAl sample at two fields. Points are experimental results and lines are predictions of Eq (3) for the parameters of fit 1 of the table. Dividing $\chi$ by the sample volume of $7 \times 10^{-5} \mathrm{~cm}^{3}$ gives $\mathrm{cgs}$ units of $\mathrm{emu} / \mathrm{cm}^{3}$.

accuracy. (2) So far susceptibility peaks of spin glasses have been found smeared $(\Delta t \geqslant 1 \%)$ in temperature, presumably because of inhomogeneity problems characteristic of these systems. On the other hand, there are reasons to expect their critical region to be broader than in ferromagnets. ${ }^{7}$ Therefore, the data on critical behavior of spin glasses comes from a wider temperature region relative to the transition temperature than in ferromagnets. In this wider temperature region, variation of the regular susceptibility is more substantial.

We expect the regular susceptibility to have a Curie-like behavior plus possible $H^{2}$ corrections. With such terms, $\chi_{\text {reg }}$ can be expanded in a series

$$
\chi_{\text {reg }}=\chi_{p}-a_{1} t-a_{2} H^{2}-a_{3} H^{2} t-a_{4} t^{2} \ldots
$$

Since the coefficients $a_{i}$ are not known a priori, one should in principle include all of them in fitting the data. Given the many parameters already needed to characterize the singular susceptibility, this is impractical. We have used $\chi_{p}$ and $a_{1}$ in conjunction with Eq. (3) to obtain the fit 1 of Table I. These parameters imply $\beta=0.7$ and $\phi=4.0$. To obtain

TABLE I. Least-squares nonlinear fits of $\chi_{\text {reg }}+\chi_{\text {sing }}$ [Eqs. (3) and (5) of text] to dc susceptibility of $7 \times 10^{-5} \mathrm{~cm}^{3}$ of $\mathrm{Gd}_{3}, \mathrm{Al}_{63}$ spin glass ${ }^{7}$ in range $15.5 \leqslant T$ $(\mathrm{K})<18,0.5<H(\mathrm{Oe})<2000$. All nonexponent units in $10^{-6} \mathrm{emu}$ (i.e., emu $/ \mathrm{cm}^{3}$ after dividing by the volume). A dash indicates this term not used in the fit. MSQD is mean square deviation, that is, the square root of chi-squared divided 240 , the number of points in the fit.

\begin{tabular}{cllll}
\hline \hline Fit No.: & 1 & 2 & 3 & 4 \\
\hline$\chi_{\text {reg }}:$ & & & & \\
$\chi_{p}$ & $6.47 \pm 0.01$ & $6.47 \pm 0.01$ & $6.47 \pm 0.01$ & $6.53 \pm 0.01$ \\
$a_{1}$ & $6.43 \pm 0.07$ & $6.38 \pm 0.08$ & $6.40 \pm 0.08$ & $7.9 \pm 0.2$ \\
$a_{2}$ & $\ldots$ & $-(6 \pm 3) \times 10^{-8}$ & $-(2 \pm 2) \times 10^{-7}$ & $\ldots$ \\
$a_{3}$ & $\ldots$ & $\ldots$ & $\ldots$ & $-8 \pm 1$ \\
$a_{4}$ & $\ldots$ & & $3.3 \pm 0.1$ & $3.2 \pm 0.1$ \\
$\chi_{\text {sing }}:$ & $3.3 \pm 0.1$ & $3.3 \pm 0.1$ & $5.6 \pm 0.1$ & $6.1 \pm 0.1$ \\
$\gamma$ & $\delta .6 \pm 0.1$ & $5.5 \pm 0.1$ & $7.1 \times 10^{-7}$ & $5.6 \times 10^{-7}$ \\
$g_{1}$ & $6.9 \times 10^{-7}$ & $7.2 \times 10^{-7}$ & $0.18 \pm 0.01$ & $0.22 \pm 0.01$ \\
$g_{2}$ & $0.19 \pm 0.01$ & $0.17 \pm 0.01$ & 0.0323 & 0.0294 \\
MSQD & 0.0323 & 0.0321 & & \\
\hline
\end{tabular}


some measure of the importance of the other terms in Eq. (5), we have done fits with each of the remaining parameters in turn, giving the fits $2-4$ in the table. We find that the mean square deviation, the critical exponents and coefficients are not significantly affected. The $-a_{1} t$ term in the regular susceptibility of Eq. (5) is the most important regular term, and in what follows we ignore the higher order terms. Since an expansion of the Curie law near $T_{G}$ gives $\chi_{p} T_{g} /\left(T_{g}-\theta\right)$ for $a_{1}$, the close similarity of $\chi_{p}$ and $a_{1}$ in the table implies $\theta=0$. Although the paramagnetic Curie temperature for our sample has previously been reported ${ }^{10}$ to be of order 30 $K$, our result is not inconsistent because of well-known deviations from the Curie law near a spin glass transition. ${ }^{11}$

The presence of the regular $-a_{1} t$ term significantly modifies our earlier conclusions about the conditions for extrema. In the limit of low field [or large $t$ in Eq. (2)] we now find a maximum at

$$
t_{\max 0}=\left(\beta g_{0} / a_{1}\right)^{1 /(1-\beta)} .
$$

Thus, surprisingly, in the $H \rightarrow 0$ limit and for nonzero $g_{0}$, the susceptibility peak is not precisely at the transition but slightly above it. In practice, however, $g_{0}$ is negligibly small and the shift is experimentally unobservable considering our temperature smearing of order $\Delta t \sim 2 \times 10^{-2}$.

At higher field, but providing $t>\left(g_{1} / g_{2}\right)^{1 / \gamma} H^{2 / \phi}$ (the crossover line), the peak position rises with a vertical tangent in the $H \cdot t$ plane and then rolls over to follow the line

$$
t=\left(\gamma g_{1} / a_{1}\right)^{1 /(\gamma+1)} H^{2 /(\gamma+1)}
$$

obtained by differentiating the $g_{1}$ and $a_{1}$ terms of Eqs. (2) and (5). Such a formula was found earlier by Bouchiat, ${ }^{12}$ but with a sign reversal which put the peak below $T_{G}$ (i.e., $t<0$ ). Plugging in the parameters of fit 1 , we find $t=0.032 H^{0.47}$, in approximate agreement with our experimental observation. However, as long as $2 /(\gamma+1)$ is less than $2 / \phi$, i.e., as long as $\beta<1$, Eq. (7) will cross the dotted crossover line as shown in the inset to Fig. 1(b). This happens at very small $t$ and $H$ for the parameters of fit 1, and at higher $H$ Eq. (7) breaks down. Direct calculation from Eq. (3) and (5) using the parameters of fit 1 gives the full contour of susceptibility maxima shown in Fig. 1(a). The temperature shift of the susceptibility maxima reaches a maximum at $t \sim 0.08$ and $H \sim 2000$ Oe. While this is a factor of 5 off in field, the overall shape of the behavior is in qualitative agreement with the experiment. The quantitative discrepancy is most likely due to approaching the high-field boundary of the critical region. Figure 2 shows a comparison of experimental and calculated $\chi(t)$ curves, with approximate agreement for the maxima even when the susceptibility peak becomes very broad. In other materials where the upward shift of the susceptibility peak has not been observed, ${ }^{6}$ we suppose that the parameters $g_{1}, g_{2}$, etc., are such as to cause the upward shift to be small and to occur at low fields.

In summary, we find that a non-mean-field scaling theory explains in a semiquantitative way the observed tendency of the equilibrium susceptibility maxima to shift first up, and then down with increasing field. Although nonequilibrium Monte Carlo calculations have shown such an effect, ${ }^{13}$ so far ours is the only equilibrium theory of spin glass behavior to do so.

The authors thank Y. Imry and K. Binder for helpful conversations. One of the authors (S.E.B.) acknowledges partial support by the National Science Foundation, Solid State Physics Grant DMR 81-20827.

${ }^{1}$ V. Cannella and J. A. Mydosh, Phys. Rev. B 6, 4220 (1976).

${ }^{2}$ B. Barbara, A. P. Malozemoff, and Y. Imry, Physica B 108, 1289 (1981).

${ }^{3}$ Y. Yeshurun (private communication).

${ }^{4}$ B. Barbara and A. P. Malozemoff, J. Less Common Metals 94, 45 (1983).

${ }^{5}$ For details on the sample, see A. P. Malozemoff, and Y. Imry, Phys. B 24, 489 (1981).

${ }^{6}$ R. V. Chamberlin, M. Hardiman, L. A. Turkevich, and R. Orbach, Phys. Rev. B 25, 6720 (1982).

'B. Barbara, A. P. Malozemoff, and Y. Imry, Phys. Rev. Lett. 47, 1852 (1981); A. P. Malozemoff, B. Barbara, and Y. Imry, J. Appl. Phys. 53, 2205 (1981).

${ }^{8}$ In Refs. 4 and 7 we used a slightly different form which, in the $t \rightarrow 0$ limit, gave a correction irregular in $t$. The mean square deviations of those earlier fits were about double those in the table of this article. The form of Eq. (3), used in this paper, avoids the irregularity in $t$ and gives a slightly better fit to the data with parameters not significantly different from those obtained earlier (see Table I).

${ }^{9}$ See Ref. 4 , where $g_{0}$ was shown to be small. In Ref. $7, g_{0}$ appeared anomalously large because the temperature dependence of the regular susceptibility was mistakenly neglected.

${ }^{10}$ T. Mizoguchi, T. R. McGuire, S. Kirkpatrick, and R. J. Gambino, Phys. Rev. Lett. 38, 89 (1977).

"A. F. J. Morgownik, J. A. Mydosh, and C. van Dijk, J. Magn. Magn. Mater. 31-34, 1334 (1983).

${ }^{12}$ H. Bouchiat, J. Phys. C 16, 445 (1983).

${ }^{13} \mathrm{~K}$. Binder and $W$. Kinzel, Proceedings of the Heidelberg Colloquium on Spin Glasses, May 30-June 2, 1983, Springer Lecture Notes (to be published). 